\title{
La Estimulación Táctil/Kinestésica Revierte los Efectos del Estrés Prenatal en la Región CA3 del Hipocampo en Ratas Hembras: Estudio Estereológico
}

\author{
Tactile/Kinesthetic Stimulation Reverses the Effects of Prenatal Stress in the \\ CA3 Region of the Hippocampus in Female Rats: A Stereological Study
}

\begin{abstract}
Walter Sepúlveda Loyola; Daniela Martínez Osorio후 German Rojas Cabezas²; Alejandro Pacheco Valles ${ }^{1}$ \& Héctor González Caro ${ }^{1}$
\end{abstract}

SEPÚLVEDA, L. W.; MARTÍNEZ, O. D.; ROJAS, C. G.; PACHECO, V.A. \& GONZÁLEZ, C. H. La estimulación táctil/kinestésica revierte los efectos del estrés prenatal en la región CA3 del hipocampo en ratas hembras: Estudio estereológico. Int. J. Morphol., 36(3):1043-1048, 2018.

RESUMEN: Esta investigación fue realizada para saber si la estimulación táctil/kinestésica postnatal es efectiva en revertir el estrés prenatal, en la citoarquitectura de CA3 del hipocampo, en crías hembras. 12 crías de ratas hembras de la cepa Sprague-Dawley, fueron distribuidas en Grupo Control (GC), Grupo con Estrés Prenatal por restricción (EP) y Grupo Estrés prenatal con Estimulación Táctil/Kinestésica postnatal (EP-ETK). El estrés prenatal en crías hembras aumentó la densidad neuronal en las áreas CA3b y CA3c $(\mathrm{p}<0,001)$. Cuando se compararon las crías con estrés prenatal con las que recibieron estimulación táctil/kinestésica temprana, disminuyeron las densidades neuronales en las áreas CA3b y CA3c, $(\mathrm{p}<0,001)$. La estimulación táctil/kinestésica postnatal logró revertir los efectos del estrés prenatal, al reducir la densidad neuronal en las áreas CA3b y CA3c del hipocampo.

PALABRAS CLAVE: Hipocampo; Estimulación táctil; Estrés prenatal; Estereología.

\section{INTRODUCCIÓN}

El estrés prenatal es motivo de investigación dirigida a comprender su estrecha relación con conductas ansiogénicas y disfunciones cognitivas observadas en la descendencia, que en hembras se revertirían hacia la adultez (Zuena et al., 2008). Estudios de estrés prenatal por restricción del movimiento de las hembras gestantes, han permitido observar en las crías consistentes incrementos de la hormona corticosterona en respuesta a estrés (Maccari et al., 2003) y atrofia en neuronas del hipocampo (Jia et al., 2010; Bock et al., 2011), ambos factores vinculados con hiperactividad del eje Hipotálamo-Pituitaria-Adrenal, HPA (Maccari et al.). La intensidad del agente estresante resulta ser fundamental en establecer las alteraciones morfológicas y funcionales identificadas en el hipocampo. De esta manera, la exposición a estrés prenatal breve y suave diario, tienen efectos beneficiosos sobre la neurogénesis y dendrogénesis, mediados por receptores mineralocorticoides; mientras que el estrés gestacional extendido y severo, tiene efectos deletéreos completamente opuestos mediados por receptores glucocorticoides (Fujioka et al., 2006). El área CA3 del hipocampo es particularmente sensibles al estrés prenatal, experimentando sus neuronas menor crecimiento de dendritas apicales y basilares durante la prepubertad (Jia et al.; Bock et al.), efectos que serían revertidos en hembras luego de aplicar manipulación o handling postnatal (Bock et al.).

El entorno perinatal se caracteriza por la presencia de estímulos maternales y sensoriales, que son determinantes para la sobrevivencia (Ronca et al., 1993) y desarrollo cognitivo de la descendencia (Liu et al., 2000; Hellstrom et al., 2012). De esta manera, los lamidos/acicalamiento proporcionados por madres cuidadoras durante la primera semana de vida, han reportado aumentar la expresión de marcadores de sinaptogénesis, sinaptofisina y N-CAM, en la adultez (Liu et al.). De manera similar, la estimulación táctil aplicada durante el periodo lactacional induce crecimiento de dendritas y de espinas dendríticas en áreas límbicas de la

${ }^{1}$ Facultad de Ciencias de la Salud, Departamento de Kinesiología, Universidad Católica del Maule, Chile.

${ }^{2}$ Universidad Tecnológica de Chile, INACAP, Chile. 
corteza prefrontal (Richards et al., 2012). En consecuencia, es posible hipotetizar que el estrés prenatal induce mecanismos que produce de atrofia celular, incrementando la densidad neuronal de la placa hipocampal; y, la estimulación táctil/kinestésica por su parte tendría efectos beneficiosos sobre el crecimiento y maduración de la placa hipocampal, que reducirían la densidad neuronal. La densidad neuronal es un marcador estereológico utilizado para identificar efectos de intervenciones postnatales sobre la corteza cerebral (Hosseini-Sharifabad \& Sabahi, 2014; González et al., 2014). Al respecto, la exposición a desnutrición calórico-proteica, aumenta la densidad neuronal en láminas supragranular e infragranular de la corteza parietal en roedores, que el ambiente enriquecido logra revertir (González et al.). Estudios con modelos de ratas autistas inducidas por ácido valproico (Edalatmanesh et al., 2013) y de esquizofrenia (HosseiniSharifabad \& Sabahi) reportan aumentos de densidad neuronal en el hipocampo, posiblemente vinculados con la exposición temprana a estrés crónico en las crías. El propósito de la presente investigación es estudiar los efectos de la estimulación táctil/kinestésica temprana en crías hembras expuestas a estrés prenatal sobre la densidad neuronal en el área CA3 del hipocampo.

\section{MATERIAL Y MÉTODO}

Se utilizaron 12 crías hembras de la cepa SpragueDawley obtenidas de 3 hembras primíparas cruzadas en laboratorios de la Universidad Católica del Maule. El día gestacional 0 , fue identificado por la presencia de tapón mucoso vaginal. Las hembras grávidas fueron asignadas a diferentes grupos experimentales. El grupo control, GC $(n=4)$, la hembras preñadas no recibieron intervención experimental durante los periodos gestacional y lactacional. El grupo estrés prenatal, $\mathrm{EP}(\mathrm{n}=4)$, recibió estrés por restricción de movimiento de la madre, de acuerdo al protocolo de Ward \& Weisz (1980). Las hembras preñadas, entre los día 14 a 20 de gestación, fueron introducidas gentilmente en un tubo estrecho de polietileno transparente de $7 \mathrm{~cm}$ de diámetro por $19 \mathrm{~cm}$ de largo y se expuso a luz brillante de 715 Watts. Este protocolo se aplicó en el ciclo nocturno del animal, cada sesión estuvo compuesta de 45 minutos y fue repetido 3 veces al día. El Grupo EP-TK (n=4), las crías fueron expuestas a estrés prenatal descrito y además recibieron estimulación táctil/kinestésica en el periodo lactacional, entre los días 3 al 12 postnatal, adaptado del protocolo de Pauk et al. (1986). La sesión de estimulación estuvo compuesta de 15 minutos: 5 minutos de estimulación táctil aplicados con un pincel suave húmedo sobre el dorso, en dirección céfalocaudal; 5 minutos de estimulación kinestésica se realizó en posición supina, con los dedos el investigador aplicó flexo- extensiones sobre los cuatro miembros; y finamente se repitió 5 minutos de estimulación táctil. Este protocolo se realizó 3 veces al día. Las camadas fueron mantenidas en condiciones controladas de temperatura $\left(22 \pm 3{ }^{\circ} \mathrm{C}\right)$, ciclo día:noche (12:12 horas) y alimentación ad libitum, hasta el día postnatal 21. Los experimentos con animales se realizaron de acuerdo a las normas de bioética de la Universidad Católica del Maule y a las normas bioéticas de adecuado manejo de animales de experimentación, de "The guide for the care and use of laboratory animals" (National Research Council, Division on Earth and Life Studies, Institute for Laboratory Animal Research, Committee for the Update of the Guide for the Care and Use of Laboratory Animals, 2011).

El sacrificio de animales se realizó el día postnatal 22. Se procedió a anestesiar por vía intraperitoneal con una dosis de pentobarbital sódico (40 mg/kg de peso corporal). Luego se realizó perfusión por vía intraventricular, inicialmente con suero fisiológico y luego con líquido de fijación Bouin (constituido por ácido pícrico $750 \mathrm{ml}, 250 \mathrm{ml}$ de formaldehido al $40 \%$ y ácido acético glacial $50 \mathrm{ml}$ ). Los cerebros extraídos fueron depositados en un recipiente hermético con líquido de fijación Bouin. Después de 48 horas de fijación, se procedió a extraer el ácido pícrico. A continuación los cerebros se incluyeron en parafina. Los tejidos fueron cortados con micrótomo en cortes coronales de 12 micrones de grosor. Posteriormente, los cortes se montaron en un portaobjeto debidamente rotulado. Finalmente, los cerebros fueron deshidratados y teñidos mediante la técnica de Nissl, con cresil violeta al 0,5\%. Las muestras se sellaron con un cubreobjeto embebido en solución Consul Mount (Thermo Scientific).

La zona de medición cerebral se estableció en la emergencia de los pies del hipocampo (Cuernos de Amon), en cortes coronales entre $-2,30 \mathrm{~mm}$ y $-3,8 \mathrm{~mm}$ del Bregma, de acuerdo con el Atlas Cerebral de Paxinos. El área CA3 del hipocampo se dividió con una línea que se proyectó entre ambos extremos del giro dentado (Brunson et al., 2001), permitiendo delimitar las zonas CA3b y CA3c. La medición de densidad neuronal (neuronas $/ \mathrm{mm}^{3}$ de hipocampo) se realizó mediante el método del Disector Óptico (Sterio, 1984; West $\&$ Gundersen, 1990). Para esto se utilizó un microscopio óptico Leica DM 1000, utilizando el objetivo 100x, bajo inmersión. Se analizaron mediciones cada 3 cortes de tejido, obteniendo 6 cortes por rata. Los criterios para el reconocimiento de neuronas se basaron en estudios previos (West \& Gundersen; González et al.). Bajo las condiciones señaladas de inmersión, se desplazó el plano focal $6 \mu \mathrm{m}$ a través de gran parte del grosor de sección del tejido. La densidad neuronal fue estimada usando la ecuación, $\mathrm{Nv}=\mathrm{Q}-/ \mathrm{h}$ x a(fra), donde Q- es el promedio total de neurona contadas, h es la altura del disector óptico $(6 \mu \mathrm{m})$ y a(fra) corresponde al área del disector $\left(2500 \mu \mathrm{m}^{2}\right)$. 
Los datos obtenidos de densidad neuronal se representaron por el promedio y la desviación estándar. En una segunda etapa se aplicó Análisis de Varianza (ANOVA) y realizó análisis de comparación múltiple de Tukey, para estudiar el efecto del tratamiento. El análisis descrito se apoyó en el programa estadístico SPSS versión 18.0. Se consideró significativo $\mathrm{p}<0,05$.

\section{RESULTADOS}

Los resultados obtenidos en la densidad neuronal de las áreas CA3b y CA3c del hipocampo se resumen en las Figuras 1 y 2, para los grupos control (C), grupo estrés prenatal (EP) y grupo estrés prenatal con estimulación táctilkinestésica (EP-ETK). En todos los casos se analizaron 4 ratas por grupo.
El área CA3b del hipocampo (Fig. 1) se observa que el grupo expuesto a estrés prenatal presentó un aumento significativo de la densidad neuronal en el día postnatal 21 (GC vs EP; a, p<0,001). La estimulación táctil/kinestésica, en tanto, redujo significativamente la densidad neuronal, respecto del grupo con estrés prenatal (EP vs EP-ETK; c, p< 0,001). Finalmente, se observaron diferencias significativas entre el grupo Control y Estrés prenatal que recibió estimulación táctil/kinestésica (GC vs EPETK; b, p<0,001). La Figura 1B, muestra una micrografía representativa del sistema de conteo celular, donde se puede observar una alta densidad neuronal en el grupo EP, que se reduce notablemente en el grupo EP-ETK .

El área CA3c del hipocampo (Fig. 2A) experimentó por efecto del estrés prenatal, aumento significativo de la densidad neuronal en el día postnatal 21 (GC vs EP; a, $\mathrm{p}<0.001)$.
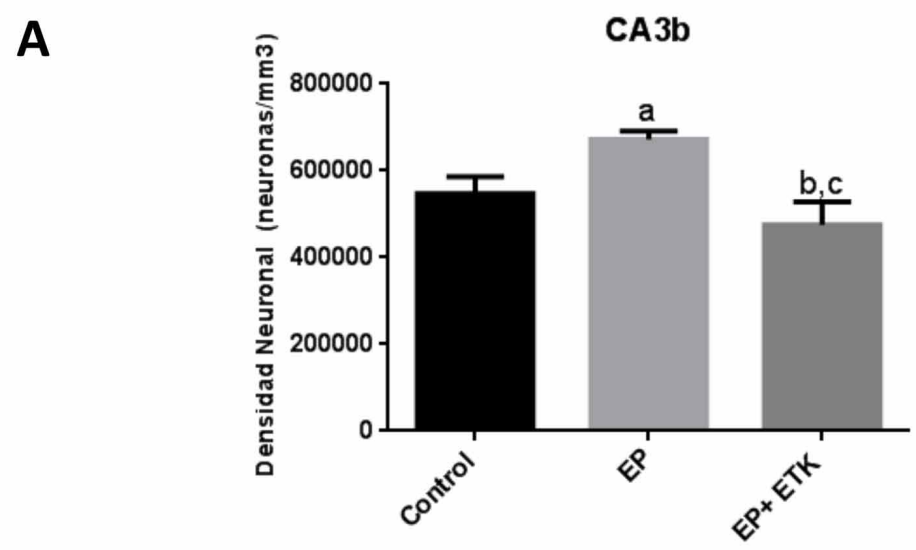

B
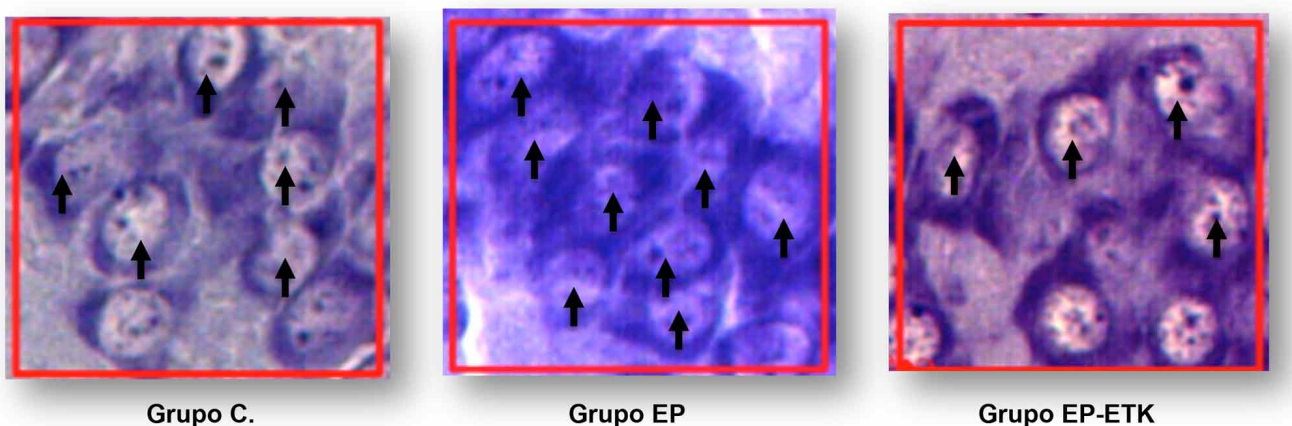

Fig. 1. Densidad Neuronal en el área CA3b del Hipocampo. (A) Gráfico que representa la densidad neuronal (promedio neuronas/mm3) de los grupos experimentales Grupo Control, Grupo EP (Grupo Estrés Prenatal) y Grupo EP-ETK (Grupo Estrés Prenatal y Estimulación Táctil-Kinestésica postnatal). Se observaron diferencias significativas entre el Grupo C vs EP (a, p<0,001); entre los grupos C vs EP-ETK (b, p<0,001) y; entre los grupos EP vs ETK., (c, p<0,001) (B) Microfotografías representativas del área CA3b del Hipocampo de los grupos C, EP y EP-ETK. La cuadrícula representa un área de $2500 \mu \mathrm{m}^{2}$. Las neuronas contabilizadas se indican con flechas negras. 
La estimulación táctil/kinestésica postnatal por su parte, indujo reducción significativa de la densidad neuronal al compararla con estrés prenatal (EP vs EP-ETK; c, p < 0,001). Por último, se observaron diferencias significativas entre el grupo control y el grupo estrés prenatal que recibió estimulación táctil/kinestésica (C vs EP-ETK; b, p<0.05). La Figura 2B, muestra una micrografía representativa del sistema de conteo celular, donde se aprecia una alta densidad neuronal en el grupo EP, que se reduce notablemente en el grupo EP-ETK.

A

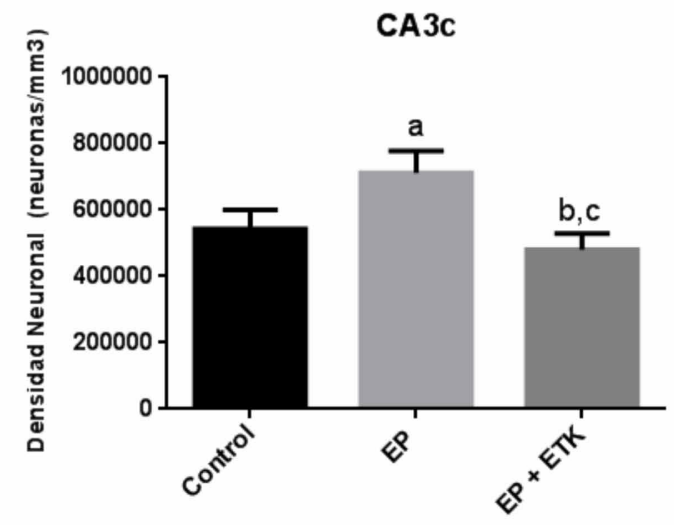

B

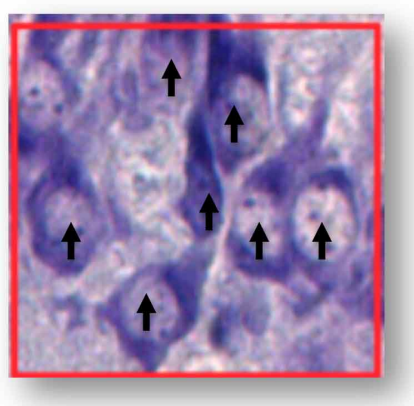

Grupo C.

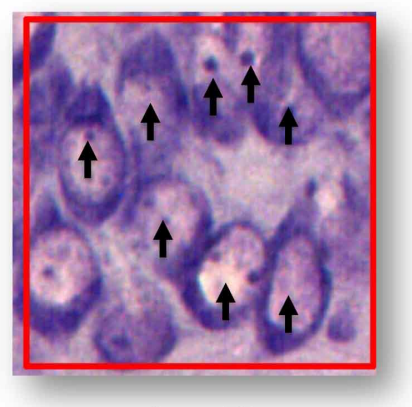

Grupo EP

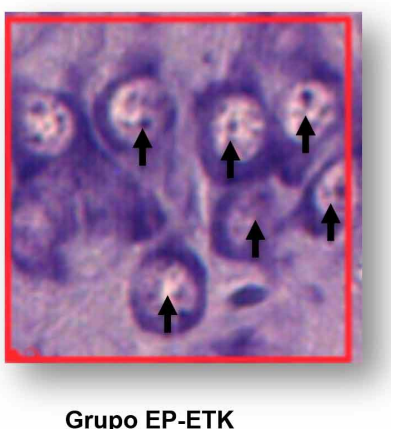

Grupo EP-ETK

Fig. 2. Densidad Neuronal en el área CA3c del Hipocampo. (A) Gráfico que representa la densidad neuronal (promedio neuronas/mm3) de los grupos experimentales Grupo C (Grupo Control), Grupo EP (Grupo Estrés Prenatal) y Grupo EP-ETK (Grupo Estrés Prenatal y Estimulación TáctilKinestésica postnatal). Se observan diferencias significativas entre el Grupo C vs EP (a, $p<0,001)$; entre los grupos C vs EP-ETK (b, p<0,05) y; entre los grupos EP vs ETK., (c, p<0,001) (B) Microfotografías representativas del área CA3c del Hipocampo de los grupos C, EP y EP-ETK. La cuadrícula representa un área de $2500 \mu \mathrm{m} 2$. Las neuronas contabilizadas se indican con flechas negras.

\section{DISCUSIÓN}

La presente investigación proporciona importante evidencia sobre los efectos deletéreos del estrés prenatal sobre la citoarquitectura de la placa hipocampal, y como la estimulación táctil/kinestésica postnatal revierte estos cambios morfológicos en crías hembras.

La exposición a estrés prenatal crónico de 2,25 horas diarias entre los días gestacionales E14-E20 del presente estudio, indujo un aumento de la densidad neuronal en áreas CA3b,c del hipocampo, el día postnatal 22 de crías hembras. Estudios similares de estrés prenatal crónico, han identificado reducción en el crecimiento dendrítico y de espinas dendríticas de células piramidales (Jia et al.; Bock et al.). Estos cambios parecen estar fuertemente inducidos por desregulación neuroendocrina de la descendencia. En primer lugar, el incremento de corticosterona inducido por estrés prenatal reduce la expresión de receptores mineralocorticoides en células granulares, que tienen directa participación con el menor crecimiento de dendritas y de espinas dendríticas (Tamura et al., 2011); y, en segundo lugar, se 
identifican incrementos de glutamato en el hipocampo que tienen un efecto dual sobre CA3, por una parte es excitotóxico debido a que incrementa el influjo de $\mathrm{Ca}^{+2}$ y reduce el crecimiento del árbol dendrítico, y por otra parte, genera una respuesta adaptativa al reducir la expresión de la subunidad NR1 del receptor NMDA, que ocasiona disfunción en tareas de aprendizaje y memoria del hipocampo (Jia et al.). Estas alteraciones moleculares tienen impacto negativo sobre las conexiones del circuito trisináptico hipocampal, generando atrofia piramidal y déficit cognitivo en la descendencia. Además, los efectos del estrés prenatal persisten hasta la adultez, observado reducción en el volumen celular y laminar de CA3 (Hosseini-Sharifabad \& Sabahi), que permitirían explicar el aumento de densidad neuronal observado en esta investigación.

Por su parte, la estimulación táctil/kinestésica (45 min diarios entre los días 3 a 12 postnatal) en crías hembras con estrés prenatal, redujo la densidad neuronal en CA3b,c. Los mecanismos involucrados de la estimulación táctil/ kinestésica, que impactan positivamente el desarrollo de neuronal del hipocampo, no son del todo conocidos. Podemos considerar dos mecanismos principales, aquellos que aumentan la complejidad del árbol dendrítico y los que actúan sobre la regulación del eje HPA, en las crías. La estimulación táctil, aplicada 10 min diarios entre los días P8-P14 de edad en crías de rata, fueron suficientes para reducir las conductas de ansiedad y mejorar en las pruebas cognitivas, las que fueron precedidas por reducción de corticosterona plasmática, aumento en la expresión de receptores glucocorticoides y aumento de BDNF en el hipocampo (Antoniazzi et al., 2017). La expresión de BDNF y BDNF mRNA en CA3 alcanza su máxima expresión en el día 14 postnatal en la rata (Das et al., 2001), participando en el crecimiento del árbol dendrítico y en la formación de espinas dendríticas en el hipocampo, dependientes de actividad neuronal (Kellner et al., 2014). Este periodo ontogenético coincide con la implementación del protocolo de estimulación tactil/kinestésica, favoreciendo los resultados observados en el presente estudio. Así también, la estimulación táctil de 15 min durante el periodo de lactancia (P3-P21) provoca cambios morfológicos en cortezas límbicas en la rata, aumentando el crecimiento dendrítico en áreas $\mathrm{Cg} 3$ y AID de la corteza frontal observadas a los dos meses de edad (Richards et al.). Estas intervenciones tempranas, si bien producen cambios relevantes en el desarrollo dendrítico, se analizan en etapas adultas, fuera del periodo crítico de lactancia, en que los cambios plásticos sobre la placa hipocampal son más evidentes.

La estimulación táctil postnatal induce cambios importantes en la regulación del eje HPA, reduciendo los niveles plasmáticos de la hormona corticosterona y aumentando la expresión del receptor glucocorticoide en el hipocampo (Jutapakdeegul et al., 2003), al compararlos con sujetos sin estimulación. Así también, la estimulación táctil breve en sujetos con estrés prenatal, aumenta la neurogénesis adulta en el giro dentado (Guerrero Aguilera et al., 2016). Estos antecedentes sugieren que la estimulación táctil ocasiona importantes beneficios en la regulación de eje HPA de estrés de las crías, en un periodo crítico de su desarrollo. En este periodo de lactancia se genera un fuerte vínculo maternaldescendencia, a través del tacto. Al respecto, la estimulación táctil/kinestésica tendría similares efectos a los lamidos/acicalamiento de madres cuidadoras, aumentando en ambos casos la expresión de glucorreceptores en el hipocampo mediado por serotonina a través de NGFI-A (Hellstrom et $a l$.), que permitiría tener una adecuada regulación del eje HPA. Por su parte, las crías que reciben alto lamidos/acicalamiento experimentaron aumento en el crecimiento dendrítico y de espinas dendríticas en el área CA1 del hipocampo en etapas adultas, al compararlas con la contraparte bajo perfil de cuidado maternal (Champagne et al., 2008).

La recuperación experimentada en CA3, producida por estimulación táctil/ kinestésica postnatal en crías con estrés prenatal, son concordantes con estudios de estimulación táctil sobre la neurogénesis adulta en el giro dentado (Guerrero Aguilera et al.) y muestran un carácter dimórfico al compararla con machos (datos no publicados), que requieren mayor investigación para esclarecer los mecanismos que explican estos cambios estructurales.

SEPÚLVEDA, L. W.; MARTÍNEZ, O. D.; ROJAS, C. G.; PACHECO, V. A. \& GONZÁLEZ, C. H. Tactile/kinesthetic stimulation reverses the effects of prenatal stress in the $\mathrm{CA} 3$ region of the hippocampus in female rats: A stereological study. Int. J. Morphol., 36(3):1043-1048, 2018.

SUMMARY: This investigation was undertaken in order to know whether the postnatal tactile/kinesthetic stimulation is effective in reversing the Prenatal Stress, in the cytoarchitecture of the $\mathrm{CA} 3$ region of the hippocampus, in female pups. 12 pups of female rats from the Sprague-Dawley strain were distributed to Control Group (GC), the Prenatal Maternal Stress by restriction group (EP) and Prenatal Maternal Stress with postnatal tactile/ kinesthetic stimulation Group (EP-ETK). The Prenatal Maternal Stress in female pups increased neuronal density in CA3b and CA3c areas $(p<0.001)$. When compared to Prenatal Maternal Stress, pups prenatal stress who received early tactile/kinesthetic stimulation showed a decrease in neuronal density in CA3b and CA3c areas ( $p$ $<0,001)$. Postnatal tactile/kinesthetic stimulation was shown to successfully reverse the Prenatal Maternal Stress effects by decreasing neuronal density in $\mathrm{CA} 3 \mathrm{~b}$ and $\mathrm{CA} 3 \mathrm{c}$ hippocampal areas.

KEY WORDS: Hippocampus; Early stimulation. Prenatal Stress; Stereology. 


\section{REFERENCIAS BIBLIOGRÁFICAS}

Antoniazzi, C. T.; Metz, V. G.; Roversi, K.; Freitas, D. L.; Vey, L. T.; Dias, V. T.; Segat, H. J.; Duarte, M. M. \& Burger, M. E. Tactile stimulation during different developmental periods modifies hippocampal BDNF and GR, affecting memory and behavior in adult rats. Hippocampus, 27(2):210-20, 2017

Bock, J.; Murmu, M. S.; Biala, Y.; Weinstock, M. \& Braun, K. Prenatal stress and neonatal handling induce sex-specific changes in dendritic complexity and dendritic spine density in hippocampal subregions of prepubertal rats. Neuroscience, 193:34-43, 2011.

Brunson, K. L.; Eghbal-Ahmadi, M.; Bender, R.; Chen, Y. \& Baram, T. Z. Long-term, progressive hippocampal cell loss and dysfunction induced by early-life administration of corticotropin-releasing hormone reproduce the effects of early-life stress. Proc. Natl. Acad. Sci. U. S. A., 98(15):8856-61, 2001.

Champagne, D. L.; Bagot, R. C.; van Hasselt, F.; Ramakers, G.; Meaney, M. J.; de Kloet, E. R.; Joëls, M. \& Krugers, H. Maternal care and hippocampal plasticity: evidence for experience-dependent structural plasticity, altered synaptic functioning, and differential responsiveness to glucocorticoids and stress. J. Neurosci., 28(23):6037-45, 2008.

Das, K. P.; Chao, S. L.; White, L. D.; Haines, W. T.; Harry, G. J.; Tilson, H. A. \& Barone, S. Jr. Differential patterns of nerve growth factor, brainderived neurotrophic factor and neurotrophin-3 mRNA and protein levels in developing regions of rat brain. Neuroscience, 103(3):73961, 2001.

Edalatmanesh, M. A.; Nikfarjam, H.; Vafaee, F. \& Moghadas, M. Increased hippocampal cell density and enhanced spatial memory in the valproic acid rat model of autism. Brain Res., 1526:15-25, 2013.

Fujioka, A.; Fujioka, T.; Ishida, Y.; Maekawa, T. \& Nakamura, S. Differential effects of prenatal stress on the morphological maturation of hippocampal neurons. Neuroscience, 141(2):907-15, 2006.

González, H.; Adaro, L.; Henrández, A. \& Fernández, V. Effects of preweaning polysensorial enrichment upon development of the parietal cortical plate of undernourished rats: A stereological study. Int. J. Morphol., 32(4):1222-7, 2014.

Guerrero Aguilera, M. A.; Rubio Osornio, M. C.; Portillo Martínez, W. \& Retana-Márquez, S. Tactile stimulation effects on hippocampal neurogenesis and spatial learning and memory in prenatally stressed rats. Brain Res. Bull., 124:1-11, 2016.

Hellstrom, I. C.; Dhir, S. K.; Diorio, J. C. \& Meaney, M. J. Maternal licking regulates hippocampal glucocorticoid receptor transcription through a thyroid hormone-serotonin-NGFI-A signalling cascade. Phil. Trans. $R$. Soc. Lond. B Biol. Sci., 367(1601):2495-510, 2012.

Hosseini-Sharifabad, M. \& Sabahi, A. Stereological analysis of cornu ammonis in prenatally stressed rats: a heuristic neurodevelopmental model of schizophrenia. Iran. J. Basic Med. Sci., 17(3):189-95, 2014.

Jia, N.; Yang, K.; Sun, Q.; Cai, Q.; Li, H.; Cheng, D.; Fan, X. \& Zhu, Z. Prenatal stress causes dendritic atrophy of pyramidal neurons in hippocampal CA 3 region by glutamate in offspring rats. Dev. Neurobiol., 70(2):114-25, 2010.

Jutapakdeegul, N.; Casalotti, S. O.; Govitrapong, P. \& Kotchabhakdi, N. Postnatal touch stimulation acutely alters corticosterone levels and glucocorticoid receptor gene expression in the neonatal rat. Dev. Neurosci., 25(1):26-33, 2003.

Kellner, Y.; Gödecke, N.; Dierkes, T.; Thieme, N.; Zagrebelsky, M. \& Korte, M. The BDNF effects on dendritic spines of mature hippocampal neurons depend on neuronal activity. Front. Synaptic Neurosci., 6:5, 2014.

Liu, D.; Diorio, J.; Day, J.; Francis, D. D. \& Meaney, M. J. Maternal care, hippocampal synaptogenesis and cognitive development in rats. Nat. Neurosci., 3(8):799-806, 2000.

Maccari, S.; Darnaudery, M.; Morley-Fletcher, S.; Zuena, A. R.; Cinque, C. \& Van Reeth, O. Prenatal stress and long-term consequences: implications of glucocorticoid hormones. Neurosci. Biobehav. Rev., 27(1-2):119-27, 2003.

National Research Council, Division on Earth and Life Studies, Institute for Laboratory Animal Research, Committee for the Update of the Guide for the Care and Use of Laboratory Animals. Guide for the Care and Use of Laboratory Animals. $8^{\mathrm{a}}$ ed. Washington D. C., The National Academies Press, 2011.

Pauk, J.; Kuhn, C. M.; Field, T. M. \& Schanberg, S. M. Positive effects of tactile versus kinesthetic or vestibular stimulation on neuroendocrine and ODC activity in maternally-deprived rat pups. Life Sci., 39(22):2081-7, 1986.

Richards, S.; Mychasiuk, R.; Kolb, B. \& Gibb, R. Tactile stimulation during development alters behaviour and neuroanatomical organization of normal rats. Behav. Brain Res., 231(1):86-91, 2012.

Ronca, A. E.; Lamkin, C. A. \& Alberts, J. R. Maternal contributions to sensory experience in the fetal and newborn rat (Rattus norvegicus). $J$. Comp. Psychol., 107(1):61-74, 1993.

Sterio, D. C. The unbiased estimation of number and sizes of arbitrary particles using the disector. J. Microsc., 134(Pt. 2):127-36, 1984.

Tamura, M.; Sajo, M.; Kakita, A.; Matsuki, N. \& Koyama, R. Prenatal stress inhibits neuronal maturation through downregulation of mineralocorticoid receptors. J. Neurosci., 31(32):11505-14, 2011.

Ward, I. L. \& Weisz, J. Maternal stress alters plasma testosterone in fetal males. Science, 207(4428):328-9, 1980.

West, M. J. \& Gundersen, H. J. Unbiased stereological estimation of the number of neurons in the human hippocampus. J. Comp. Neurol., 296(1):1-22, 1990.

Zuena, A. R.; Mairesse, J.; Casolini, P.; Cinque, C.; Alemà, G. S.; MorleyFletcher, S.; Chiodi, V.; Spagnoli, L. G.; Gradini, R.; Catalani, A.; Nicoletti, F. \& Maccari, S. Prenatal restraint stress generates two distinct behavioral and neurochemical profiles in male and female rats. PLoS One, 3(5):e2170, 2008.

\section{Dirección para correspondencia \\ MSc Héctor González Caro \\ Facultad de Ciencias de la Salud \\ Departamento de Kinesiología \\ Universidad Católica del Maule \\ San Miguel 3605 \\ Talca \\ CHILE}

\section{Email: hgonzale@ucm.cl}

Recibido : 16-11-2017

Aceptado: 24-04-2018 